\title{
Dirac Field as a Source of the Inflation in $2+1$ Dimensional Teleparallel Gravity
}

\author{
Ganim Gecim and Yusuf Sucu \\ Department of Physics, Faculty of Science, Akdeniz University, 07058 Antalya, Turkey \\ Correspondence should be addressed to Yusuf Sucu; ysucu@akdeniz.edu.tr
}

Received 2 November 2016; Revised 2 January 2017; Accepted 4 January 2017; Published 31 January 2017

Academic Editor: Shi-Hai Dong

Copyright () 2017 Ganim Gecim and Yusuf Sucu. This is an open access article distributed under the Creative Commons Attribution License, which permits unrestricted use, distribution, and reproduction in any medium, provided the original work is properly cited. The publication of this article was funded by SCOAP ${ }^{3}$.

\begin{abstract}
In this paper, we study early-time inflation and late-time acceleration of the universe by nonminimally coupling the Dirac field with torsion in the spatially flat Friedman-Robertson-Walker (FRW) cosmological model background. The results obtained by the Noether symmetry approach with and without a gauge term are compared. Additionally, we compare these results with that of the $3+1$ dimensional teleparallel gravity under Noether symmetry approach. And we see that the study explains early-time inflation and late-time acceleration of the universe.
\end{abstract}

\section{Introduction}

The recent cosmological observational data point out that our universe has two acceleration periods: early-time and late-time cosmic acceleration. Some cosmological difficulties, such as the initial singularity problem, the flatness problem, and the horizon problem, are resolved by using the early-time inflation scenario with a scalar field as a source of quantum fluctuations $[1,2]$. On the other hand, the late-time inflation scenario is due to the observed accelerating expansion of the present universe [3-5], where the acceleration is explained with a negative pressure component which is called dark energy. The models including different dark energy forms, such as cosmological constant $[6,7]$, canonical scalar field (i.e., quintessence) $[8,9]$, and a phantom field $[10,11]$, have been discussed widely in the context of the standard Einstein relativity.

The teleparallel gravity is one of the modified theories which has an alternative geometrical formulation of the Einstein's general relativity. It was firstly introduced by Einstein to merge the gravitation with electromagnetism [12]. Though the space-time structure in the standard Einstein general relativity is described by a Levi-Civita connection which has a free-torsion, but nonvanishing curvature, the space-time structure in the teleparallel gravity is constructed by a Weitzenbock connection which is characterized by the metricity condition and vanishing curvature, but it has torsion [13]. Also, differently from the standard Einstein relativity, the teleparallel gravity is a gauge theory of the space-time translation group [14-17].

In recent decades, the $2+1$ dimensional gravitation theories, such as the standard Einstein general relativity $[18,19]$, the new massive gravity [20-22], the topological massive gravity [23-26], and the teleparallel gravity [2729], have attracted considerable attention in the literature. In particular, the $2+1$ dimensional teleparallel theory covers an important place among the $2+1$ dimensional gravity theories because it has a Newtonian limit, a black hole solution [27], and a gravitational wave solution named Siklos waves that are a special class of the exact gravitational waves propagating on the AdS background [30]. Also, the other black hole solutions are performed in the context of the $2+1$ dimensional teleparallel gravity [31], $f(T)$ gravity [32], Maxwell- $f(T)$ gravity [33], and torsion scalar coupled with a scalar field nonminimally [34].

As the Dirac theory has a vacuum including Zitterbewegung oscillations between positive and negative energy states and perfectly explains an interaction of a Dirac particle with an atomic structure of the materials, it gives a reasonable cosmological solutions for the early-time inflation and 
late-time acceleration of the universe [35]. Therefore, in this study, we nonminimally couple the Dirac field with the torsion scalar and consider the Dirac field as a source of earlytime inflation and late-time acceleration in the context of the $2+1$ dimensional teleparallel gravity on the FriedmannRobertson-Walker (FRW) background by using the Noether gauge symmetry approach. At same time, we show that the cosmological solutions obtained in the context of the $2+$ 1 dimensional teleparallel gravity are compatible with the results which are in the coupling the Dirac field with the $3+1$ dimensional gravity theories [36-38] and the $2+1$ dimensional Einstein gravity [35].

This paper is organized as follows. In the following section, we find the field equations in which the Dirac field is nonminimal coupled with the $2+1$ dimensional teleparallel gravity. In Section 3, we search the Noether symmetry for the Lagrangian which is coupled Dirac field with the $2+1$ dimensional teleparallel gravity. In Section 4, we obtain solutions of the field equations by using Noether symmetry approach. Furthermore, we find solutions of the field equations under the zero-gauge term condition and compare these results with the results of study performed in the $3+1$ dimensional teleparallel gravity. Finally, in Section 5, we conclude with a summary of the obtained results. Throughout the paper, we use $c=G=\hbar=1$.

\section{The Field Equations}

The investigations on the interaction between a torsion and a Dirac spinorial field have attracted attention for a long time [39-48]. The main purpose of these studies is to construct modified gravitational theories that include a spin effect of the matter fields naturally. Therefore, to consider the inflationary problem of the universe in the framework of the $2+1$ dimensional teleparallel gravity nonminimally coupled with Dirac field, we start by writing the action nonminimally coupled a Dirac field with a torsion scalar as follows:

$$
\begin{aligned}
\mathscr{A} & =\int d^{3} x|e|\{F(\Psi) T \\
& \left.+\frac{\imath}{2}\left[\bar{\psi} \sigma^{\mu}(x)\left(D_{\mu} \psi\right)-\left(D_{\mu} \bar{\psi}\right) \sigma^{\mu}(x) \psi\right]-V(\Psi)\right\},
\end{aligned}
$$

where $F(\Psi)$ and $V(\Psi)$ are the coupling functions and the self-interaction potential, respectively. These scalar functions depend on only function of a bilinear Dirac field $\Psi=$ $\bar{\psi} \psi$. And the bilinear Dirac field $\Psi$ is a relativistic invariant quantity [49], where $\bar{\psi}$ is the adjoint of the Dirac field $\psi$, which $\bar{\psi}=\psi^{\dagger} \sigma^{3}$. In this action, $|e|=\operatorname{det}\left(e^{(i)}{ }_{\mu}(x)\right)=\sqrt{|g|}$, where $e^{(i)}{ }_{\mu}(x)$ are dreibeins and $g$ is determinant of the metric tensor, $g_{\mu \nu}$, defined in terms of the dreibeins and the $2+1$ dimensional Minkowski space-time metric, $\eta_{(i)(j)}$, with the signature $(1,-1,-1)$, as $g_{\mu \nu}=e^{(i)} e_{\mu}^{(j)}{ }_{\nu} \eta_{(i)(j)}$, where $i$ and $j$ are the internal (local) space-time indices, and $\mu$ and $\nu$ are the external (global) space-time indices $[50,51] . \sigma^{\mu}(x)$ are the Dirac matrices dependent on space-time coordinates, and they are defined in terms of the flat space-time Dirac matrices, $\bar{\sigma}^{i}$, and the dreibeins as follows:

$$
\sigma^{\mu}(x)=e_{(i)}^{\mu}(x) \bar{\sigma}^{i}
$$

where $\bar{\sigma}^{i}$ are

$$
\begin{aligned}
& \bar{\sigma}^{0}=\sigma^{3}, \\
& \bar{\sigma}^{1}=\imath \sigma^{1}, \\
& \bar{\sigma}^{2}=\imath \sigma^{2}
\end{aligned}
$$

and $\imath$ is the imaginary unit (i.e., $\iota^{2}=-1$ ), and $\sigma^{1}, \sigma^{2}$ and $\sigma^{3}$ are the standard $2 \times 2$ Pauli matrices $[50,52] . T$ is the Weitzenbock invariant (i.e., torsion scalar) defined as $[32,33]$

$$
T=S_{\rho}^{\mu \nu} T_{\mu \nu}^{\rho},
$$

where $T_{\mu \nu}^{\rho}$ is the Weitzenbock torsion tensor defined in terms of the Weitzenbock connection $[13,32],{ }^{W} \Gamma_{\mu \nu}{ }_{\mu \nu}=$ $e_{(i)}^{\rho} \partial_{\nu} e^{(i)}$,

$$
T_{\mu \nu}^{\rho}=e_{(i)}^{\rho}\left[\partial_{\mu} e_{\nu}^{(i)}-\partial_{\nu} e_{\mu}^{(i)}\right]
$$

and $S_{\rho}{ }^{\mu \nu}$ is a skew-symmetric tensor defined as

$$
\begin{aligned}
S_{\rho}^{\mu \nu}= & \frac{1}{4}\left[T_{\rho}^{\nu \mu}-T_{\rho}^{\mu \nu}+T_{\rho}^{\mu \nu}\right] \\
& +\frac{1}{2}\left[\delta_{\rho}^{\mu} T_{\beta}^{\beta \nu}-\delta_{\rho}^{\nu} T_{\beta}^{\beta \mu}\right] .
\end{aligned}
$$

Furthermore, the torsion tensor can be decomposed into three irreducible parts in the $2+1$ dimensional spacetime under the $S O(2,1)$ group $[53,54]$ : the trace part, $t_{\mu}=T_{\mu \nu}{ }^{\nu}$, the totally antisymmetric part (i.e., pseudoscalar), $\chi=(1 / 6) \varepsilon^{\mu \nu \lambda} T_{\mu \nu \lambda}$, and the traceless symmetric tensor, $X_{\mu \nu}$. Hence, the torsion tensor is defined in terms of these components as follows:

$$
T_{\mu \nu \lambda}=\chi \varepsilon_{\mu \nu \lambda}+\frac{1}{2}\left(\eta_{\nu \lambda} t_{\mu}-\eta_{\mu \lambda} t_{\nu}\right)+\varepsilon_{\mu \nu \sigma} X_{\lambda}^{\sigma}
$$

where $\varepsilon_{\mu v \sigma}$ is the three-dimensional Levi-Civita symbol $\left(\varepsilon_{012}=1\right)$. Accordingly, in a $2+1$ dimensional spacetime, the minimal coupling between torsion and Dirac field is described by the totally antisymmetric part of the torsion tensor which is pseudoscalar $[53,54]$. On the other hand, the Weitzenbock connection can be decomposed as ${ }^{W} \Gamma_{\mu \nu}^{\rho}=$ $\Gamma_{\mu \nu}^{\rho}+K_{\mu \nu}^{\rho}$, where $\Gamma_{\mu \nu}^{\rho}$ is the Christoffel connection and $K_{\mu \nu}^{\rho}$ is the contorsion tensor, $K^{(i)}{ }_{(j) \mu}=(1 / 2) e^{(i)}{ }_{\beta} e_{(j)}{ }^{\nu}\left(T_{\nu}{ }_{\mu}{ }_{\mu}+T_{\mu}{ }^{\beta}{ }_{\nu}-\right.$ $T_{\mu \nu}^{\beta}$ ) [43]. Also, the relationship between the Weitzenbock spin connection, $\omega^{(i)}(j) \mu$, and general relativity (Lorentz) spin connection, ${ }^{0} \omega^{(i)}{ }_{(j) \mu}$, becomes as $\omega^{(i)}{ }_{(j) \mu}={ }^{0} \omega^{(i)}{ }_{(j) \mu}+K^{(i)}{ }_{(j) \mu}$. As the teleparallel gravity is characterized by the vanishing Weitzenbock spin connection (i.e., $\omega^{(i)}{ }_{(j) \mu}={ }^{0} \omega^{(i)}{ }_{(j) \mu}+K^{(i)}{ }_{(j) \mu}$ $=0)[43,44,48]$, the covariant derivative of the Dirac spinor, 
$D_{\mu} \psi$, and its adjoint, $D_{\mu} \bar{\psi}$, can be expressed, respectively, as $[48,53]$

$$
\begin{aligned}
& D_{\mu} \psi=\partial_{\mu} \psi-\frac{\imath}{4}\left({ }^{0} \omega^{\left({ }^{(i)}\right.}{ }_{(j) \mu}\right) s_{(i)}{ }^{(j)} \psi, \\
& D_{\mu} \bar{\psi}=\partial_{\mu} \bar{\psi}+\frac{\imath}{4}\left({ }^{0} \omega^{(i)}{ }_{(j) \mu}\right) s_{(i)}{ }^{(j)} \bar{\psi},
\end{aligned}
$$

where $s_{(i)}{ }^{(j)}$ is a spin operator and it is defined in terms of Dirac matrices: $s_{(i)}{ }^{(j)}=(\imath / 2)\left[\bar{\sigma}_{(i)}, \bar{\sigma}^{(j)}\right]$. Furthermore, under these considerations, the minimal coupling of the Dirac spinor to the torsion becomes equivalent to the that of curvature. Accordingly, the Dirac spinor can be consistently coupled to torsion as indicated in previously works [48, 5557].

The variation of action (1) with respect to $\bar{\psi}$ and $\psi$ gives us the Dirac equation and its adjoint as follows:

$$
\begin{aligned}
\imath \sigma^{\mu}(x) D_{\mu} \psi+\left[F^{\prime} T-V^{\prime}\right] \psi & =0, \\
\imath\left(D_{\mu} \bar{\psi}\right) \sigma^{\mu}(x)-\left[F^{\prime} T-V^{\prime}\right] \bar{\psi} & =0,
\end{aligned}
$$

respectively, where the prime denotes the derivative with respect to the bilinear $\Psi$. Additionally, the variation of the action with respect to the dreibein fields, $e^{(i)}{ }_{\sigma}(x)$, yields the equation of motion as follows:

$$
\begin{gathered}
F\left[\operatorname{Te}_{(i)}{ }^{\sigma}-4 T^{\alpha}{ }_{\nu(i)} S_{\alpha}{ }^{\nu \sigma}+4 e^{-1} \partial_{\alpha}\left(e S_{(i)}{ }^{\alpha \sigma}\right)\right] \\
+4 F^{\prime}\left(\partial_{\alpha} \Psi\right) S_{(i)}{ }^{\alpha \sigma}=\mathrm{T}_{(i)}{ }^{\sigma},
\end{gathered}
$$

where $T^{\alpha}{ }_{\nu(i)}=e_{(i)}{ }^{\lambda} T^{\alpha}{ }_{\nu \lambda}$ and $S_{(i)}{ }^{\alpha \sigma}=e_{(i)}{ }^{\lambda} S_{\lambda}{ }^{\alpha \sigma}$ and $\mathrm{T}_{(i)}{ }^{\sigma}$ is the modified energy-momentum tensor of the Dirac field and its explicit form is expressed as

$$
\begin{aligned}
\mathrm{T}_{\beta \mu}= & \frac{\imath}{2}\left[\bar{\psi} \sigma_{\beta}(x) D_{\mu} \psi-\left(D_{\mu} \bar{\psi}\right) \sigma_{\beta}(x) \psi\right] \\
& +g_{\beta \mu}\left[\left(F^{\prime} T-V^{\prime}\right) \Psi+V\right] .
\end{aligned}
$$

To investigate the inflationary problem in the context of the $2+1$ dimensional teleparallel gravity, we will consider the $2+1$ dimensional FRW space-time background that is spatially flat, homogeneous, and isotropic universe:

$$
d s^{2}=d t^{2}-a^{2}(t)\left[d x^{2}+d y^{2}\right]
$$

where $a(t)$ is the scale factor of the universe. For the metric, the dreibein fields and their dual are expressed as $e^{(i)}{ }_{\mu}=\operatorname{diag}(1, a(t), a(t))$ and $e_{(i)}{ }^{\mu}=\operatorname{diag}(1,1 / a(t), 1 / a(t))$, respectively. Accordingly, the nonnull components of the torsion tensor (5) and the $S_{\rho}{ }^{\mu \nu}$ skew-symmetric tensor (6) are given by, respectively,

$$
\begin{aligned}
& T_{01}^{1}=T_{02}^{2}=H, \\
& S_{1}{ }^{10}=S_{2}{ }^{20}=\frac{H}{2},
\end{aligned}
$$

where $H=\dot{a} / a$ is the Hubble parameter and the dot represents differentiation with respect to cosmic time, $t$. Moreover, using (4), the torsion scalar becomes $T=-2 H^{2}$. Hence, the Dirac equation (9) and its adjoint (10) are reduced as, respectively,

$$
\begin{aligned}
& \dot{\psi}+H \psi+\imath V^{\prime} \sigma^{3} \psi+2 \imath H^{2} F^{\prime} \sigma^{3} \psi=0, \\
& \dot{\bar{\psi}}+H \bar{\psi}-\imath V^{\prime} \bar{\psi} \sigma^{3}-2 \imath H^{2} F^{\prime} \bar{\psi} \sigma^{3}=0 .
\end{aligned}
$$

Furthermore, the " 00 " and " 11 " components of the equation of motion (11) give us the following:

$$
\begin{aligned}
H^{2} & =\frac{V}{2 F}, \\
\frac{\ddot{a}}{a} & =-\frac{2 H F^{\prime} \dot{\Psi}+\left[2 H^{2} F^{\prime}+V^{\prime}\right] \Psi-V}{2 F},
\end{aligned}
$$

respectively. Also, the "22" component is equivalent to the "11" component.

In order to solve the field equations (17) and (18), suitable forms of the unknown functions $F(\Psi)$ and $V(\Psi)$ must be given. However, in this study, we calculate the forms of these functions by using the Noether gauge symmetry approach method. For this reason, firstly, we must construct the Lagrangian of the system defined by (1). Therefore, with similar calculations as in [35], the point-like Lagrangian of the system can be written from action (1) in the following form:

$$
L=2 F \dot{a}^{2}-\frac{\imath a^{2}}{2}\left(\bar{\psi} \sigma^{3} \dot{\psi}-\dot{\bar{\psi}} \sigma^{3} \psi\right)+a^{2} V .
$$

Dirac's equations for the spinor field $\psi$ and its adjoint $\bar{\psi}$ are obtained from the Lagrangian (19) such that the EulerLagrange equations of $\psi$ and $\bar{\psi}$ are, respectively,

$$
\begin{aligned}
& \dot{\psi}+H \psi+\imath V^{\prime} \sigma^{3} \psi+2 \imath H^{2} F^{\prime} \sigma^{3} \psi=0, \\
& \dot{\bar{\psi}}+H \bar{\psi}-\imath V^{\prime} \bar{\psi} \sigma^{3}-2 \imath H^{2} F^{\prime} \bar{\psi} \sigma^{3}=0 .
\end{aligned}
$$

Furthermore, using the point-like Lagrangian (19) and Dirac's equations, we find the second-order Euler-Lagrange equation for $a$, that is, the acceleration equation,

$$
\frac{\ddot{a}}{a}=-\frac{p_{f}}{2 F},
$$

where $p_{f}$,

$$
p_{f}=2 H F^{\prime} \dot{\Psi}+\left[2 H^{2} F^{\prime}+V^{\prime}\right] \Psi-V,
$$

is the pressure of the Dirac field. Furthermore, the constraint equation for the energy function $\left(E_{L}=0\right)$ associated with the point-like Lagrangian (19) is written as

$$
E_{L}=\frac{\partial L}{\partial \dot{a}} \dot{a}+\frac{\partial L}{\partial \dot{\psi}} \dot{\psi}+\dot{\bar{\psi}} \frac{\partial L}{\partial \dot{\bar{\psi}}}-L,
$$

$[58,59]$, which is equivalent to the Friedmann equation:

$$
H^{2}=\frac{\rho_{f}}{2 F},
$$


where $\rho_{f}$ represents the effective energy density and it is given by the self-interaction potential of a Dirac field in the following form:

$$
\rho_{f}=V
$$

As can be seen, the Dirac equation (15) and its adjoint (16) as well as the equations of motion (17) and (18) were recovered again by using the Lagrangian formalism.

\section{The Noether Symmetry Approach}

The symmetry conception has always played a central role in physics because it is directly associated with the conservation laws of a dynamical system [60]. These connections between symmetries and conservation laws of a dynamical system are expressed by the Noether's theorem [61]. So, in this study, the existence of Noether symmetries led to a specific form of coupling function and the self-interaction potential, which are important for obtaining the exact solutions of the fields equations [58, 59, 62-66]. Mathematically, the Noether symmetry condition for the system where the Dirac field is coupled with the $2+1$ dimensional gravity, with a gauge term, $B$, can be expressed as follows [35, 63, 67-69]:

$$
\mathbf{X}^{[1]} L+L\left(D_{t} \tau\right)=D_{t} B
$$

where $B=B\left(t, a, \psi_{j}, \psi_{j}^{\dagger}, \dot{a}, \dot{\psi}_{j}, \dot{\psi}_{j}^{\dagger}\right)$ is a gauge term and $D_{t}$ is the operator of the total differentiation with respect to $t$

$$
D_{t}=\frac{\partial}{\partial t}+\dot{a} \frac{\partial}{\partial a}+\sum_{j=1}^{2}\left(\dot{\psi}_{j} \frac{\partial}{\partial \psi_{j}}+\dot{\psi}_{j}^{\dagger} \frac{\partial}{\partial \psi_{j}^{\dagger}}\right),
$$

and $\mathbf{X}^{[1]}$, where

$$
\begin{aligned}
\mathbf{X}^{[1]}= & \mathbf{X}+\left(D_{t} \alpha-\dot{a} D_{t} \tau\right) \frac{\partial}{\partial \dot{a}} \\
& +\sum_{j=1}^{2}\left[\left(D_{t} \beta_{j}-\dot{\psi}_{j} D_{t} \tau\right) \frac{\partial}{\partial \dot{\psi}_{j}}\right] \\
& +\sum_{j=1}^{2}\left[\left(D_{t} \gamma_{j}-\dot{\psi_{j}^{\dagger}} D_{t} \tau\right) \frac{\partial}{\partial \dot{\dot{\psi}_{j}^{\dagger}}}\right],
\end{aligned}
$$

is the first-order prolongation of the vector field $\mathbf{X}$ given by

$$
\mathbf{X}=\tau \frac{\partial}{\partial t}+\alpha \frac{\partial}{\partial a}+\sum_{j=1}^{2}\left(\beta_{j} \frac{\partial}{\partial \psi_{j}}+\gamma_{j} \frac{\partial}{\partial \psi_{j}^{\dagger}}\right),
$$

where coefficients $\tau, \alpha, \beta_{j}$, and $\gamma_{j}$ are dependent on variables $t$, $a, \psi_{j}, \psi_{j}^{\dagger}, \dot{a}, \dot{\psi}_{j}$, and $\dot{\psi}_{j}^{\dagger}$. Letting the spinor field $\psi=\left(\psi_{1}, \psi_{2}\right)^{T}$ and its adjoint $\bar{\psi}=\psi^{\dagger} \sigma^{3}$, the point-like Lagrangian (19) is reduced to the following form:

$$
L=2 F \dot{a}^{2}-\frac{\imath a^{2}}{2}\left[\sum_{j}^{2}\left(\psi_{j}^{\dagger} \dot{\psi}_{j}-\dot{\psi}_{j}^{\dagger} \psi_{j}\right)\right]+a^{2} V .
$$

Hence, the point-like Lagrangian (31) and the Noether gauge symmetry condition (27) lead to the following partial differential equations obtained from the fact that the coefficients of $\dot{a}^{3}, \dot{a}^{2}, \dot{a}, \dot{\psi}_{j}, \dot{\psi}_{j}^{\dagger}, \dot{a} \dot{\psi}_{j}, \dot{a} \dot{\psi}_{j}^{\dagger}, \dot{a}^{2} \dot{\psi}_{j}$, and $\dot{a} \dot{\psi}_{j}^{\dagger}$ vanish, separately:

$$
\begin{aligned}
& 2 F\left(2 \frac{\partial \alpha}{\partial a}-\frac{\partial \tau}{\partial t}\right)+2 F^{\prime} \sum_{j=1}^{2} \epsilon_{j}\left(\beta_{j} \psi_{j}^{\dagger}+\gamma_{j} \psi_{j}\right)=0, \\
& 4 F \frac{\partial \alpha}{\partial \psi_{j}}=0 \\
& 4 F \frac{\partial \alpha}{\partial \psi_{j}^{\dagger}}=0 \\
& 2 F \frac{\partial \tau}{\partial \psi_{j}}=0 \\
& 2 F \frac{\partial \tau}{\partial \psi_{j}^{\dagger}}=0 \\
& 2 F \frac{\partial \tau}{\partial a}=0, \\
& \imath \alpha \psi_{j}+\frac{\imath a}{2} \beta_{j}-\frac{\imath a}{2} \sum_{i=1}^{2}\left(\frac{\partial \beta_{i}}{\partial \psi_{j}^{\dagger}} \psi_{i}^{\dagger}-\frac{\partial \gamma_{i}}{\partial \psi_{j}^{\dagger}} \psi_{i}\right)+a V \frac{\partial \tau}{\partial \psi_{j}^{\dagger}} \\
& -\frac{1}{a} \frac{\partial B}{\partial \psi_{j}^{\dagger}}=0 \\
& \imath \alpha \psi_{j}^{\dagger}+\frac{\imath a}{2} \gamma_{j}+\frac{\imath a}{2} \sum_{i=1}^{2}\left(\frac{\partial \beta_{i}}{\partial \psi_{j}} \psi_{i}^{\dagger}-\frac{\partial \gamma_{i}}{\partial \psi_{j}} \psi_{i}\right)-a V \frac{\partial \tau}{\partial \psi_{j}} \\
& +\frac{1}{a} \frac{\partial B}{\partial \psi_{j}}=0 \\
& 4 F \frac{\partial \alpha}{\partial t}-\frac{\imath a^{2}}{2} \sum_{j=1}^{2}\left(\frac{\partial \beta_{j}}{\partial a} \psi_{j}^{\dagger}-\frac{\partial \gamma_{j}}{\partial a} \psi_{j}\right)+a^{2} V \frac{\partial \tau}{\partial a} \\
& -\frac{\partial B}{\partial a}=0
\end{aligned}
$$

and, also, the remaining expressions satisfy the following equation:

$$
\begin{aligned}
(2 \alpha & \left.+a \frac{\partial \tau}{\partial t}\right) V-\frac{1}{a} \frac{\partial B}{\partial t}+a V^{\prime} \sum_{j=1}^{2} \epsilon_{j}\left(\beta_{j} \psi_{j}^{\dagger}+\gamma_{j} \psi_{j}\right) \\
& -\frac{\imath a}{2} \sum_{j=1}^{2}\left(\psi_{j}^{\dagger} \frac{\partial \beta_{j}}{\partial t}-\psi_{j} \frac{\partial \gamma_{j}}{\partial t}\right)=0
\end{aligned}
$$

where

$$
\epsilon_{j}= \begin{cases}1 & \text { for } j=1 \\ -1 & \text { for } j=2\end{cases}
$$


Then, the complete solutions of (32) are obtained as follows:

$$
\begin{aligned}
\alpha & =-\frac{c_{1}(k+1)}{2(k-1)} a, \\
\beta_{j} & =\frac{c_{1}(k+1)}{2(k-1)} \psi_{j}+\epsilon_{j} \beta_{0} \psi_{j}, \\
\gamma_{j} & =\frac{c_{1}(k+1)}{2(k-1)} \psi_{j}^{\dagger}-\epsilon_{j} \beta_{0} \psi_{j}^{\dagger}, \\
\tau & =c_{1} t+c_{2}, \\
B & =c_{4}
\end{aligned}
$$

and the coupling function, $F(\Psi)$, is found as

$$
F(\Psi)=f_{0} \Psi^{2 k /(k+1)},
$$

where $c_{1}, c_{2}, c_{4}, f_{0}$, and $k(k \neq 1)$ are integration constants. Finally, inserting (35) and (36) in (33), the self-interaction potential is determined as

$$
V(\Psi)=\lambda \Psi^{2 /(k+1)}
$$

where $\lambda$ is integration constants.

Using (35), the corresponding Noether gauge symmetry generators are obtained as

$$
\begin{aligned}
\mathbf{X}_{0}= & \frac{\partial}{\partial t}, \\
\mathbf{X}_{1}= & t \frac{\partial}{\partial t} \\
& -\frac{k+1}{2(k-1)}\left[a \frac{\partial}{\partial a}-\sum_{i=1}^{2}\left(\psi_{i} \frac{\partial}{\partial \psi_{i}}+\psi_{i}^{\dagger} \frac{\partial}{\partial \psi_{i}^{\dagger}}\right)\right], \\
\mathbf{X}_{2}= & \sum_{i=1}^{2} \epsilon_{i}\left(\psi_{i} \frac{\partial}{\partial \psi_{i}}-\psi_{i}^{\dagger} \frac{\partial}{\partial \psi_{i}^{\dagger}}\right) .
\end{aligned}
$$

Furthermore, these generators satisfy the following commutation relations:

$$
\begin{aligned}
& {\left[\mathbf{X}_{0}, \mathbf{X}_{1}\right]=\mathbf{X}_{0},} \\
& {\left[\mathbf{X}_{0}, \mathbf{X}_{2}\right]=\left[\mathbf{X}_{1}, \mathbf{X}_{2}\right]=0 .}
\end{aligned}
$$

Thanks to the Noether theorem, if vector field $\mathbf{X}$ is a Noether gauge symmetry corresponding to the Lagrangian $L$, then the following equivalent is a first integral (i.e., conserved quantities of the system) associated with $\mathbf{X}$ :

$$
\begin{aligned}
I= & \tau L+(\alpha-\tau \dot{a}) \frac{\partial L}{\partial \dot{a}}+\sum_{j=1}^{2}\left[\left(\beta_{j}-\tau \dot{\psi}_{j}\right) \frac{\partial L}{\partial \dot{\psi}_{j}}\right] \\
& +\sum_{j=1}^{2}\left[\left(\gamma_{j}-\tau \dot{\psi} \dot{j}\right) \frac{\partial L}{\partial \dot{\psi}_{j}^{\dagger}}\right]-B .
\end{aligned}
$$

Moreover, there are three first integrals (or conserved quantities) associated with the Noether gauge symmetries:

$$
\begin{aligned}
& I_{0}=-2 F \dot{a}^{2}+a^{2} V, \\
& I_{1}=t I_{0}-\frac{2 F(k+1)}{(k-1)} a \dot{a}, \\
& I_{2}=-\frac{\imath a^{2} \Psi}{2} .
\end{aligned}
$$

It is important to note that the first integral (41) is associated with the energy function (24), since the first integral $I_{0}$ vanishes identically.

\section{The Solutions of the Field Equations}

To determine the time evolution of the scale factor, $a(t)$, firstly, we need to know the form of bilinear function, $\Psi$. Thus, we insert the coupling function $F$ given by (36) in the Dirac equation (20) and in its adjoint (21) and we get

$$
\dot{\Psi}+2 \frac{\dot{a}}{a} \Psi=0
$$

Then, the form of the bilinear function is calculated as

$$
\Psi=\frac{\Psi_{0}}{a^{2}},
$$

where $\Psi_{0}$ is a constant of integration. Then, the first integral equation (43) is found $I_{2}=-\imath \Psi_{0} / 2$. Furthermore, inserting (45) and the coupling function (36) in the first integral (42), we get

$$
\dot{a}+K a^{(3 k-1) /(k+1)}=0
$$

where

$$
K=\frac{(k-1) I_{1}}{2(k+1) f_{0} \Psi_{0}^{2 k /(k+1)}},
$$

and then integrating this equation, the scale factor $a(t)$ is found as

$$
a(t)=\left[\frac{2 K(k-1)}{k+1} t+a_{0}\right]^{(k+1) / 2(1-k)},
$$

where $a_{0}$ is an integration constant. Using the solution in (48) in the acceleration equation (22) and in the Friedmann equation (25), we obtain constraint relations between the constants as $\lambda=I_{1}^{2}(k-1)^{2} / 2 \Psi_{0}^{2} f_{0}(k+1)^{2}$. Under these conditions, the scale factor that is obtained from the Noether gauge symmetry represents a power law expansion for the universe. Moreover, using the definition of the deceleration parameter $q$, namely, $q=-a \ddot{a} / \dot{a}^{2}$, it can be seen that the evolution of the universe has undergone three different processes. Using (48), the deceleration parameter is calculated as $q=(1-3 k) /(k+1)$. From the deceleration parameter and (48), we see that the scale factor, $a(t)$, is up to parameter $k$, and it corresponds to these different cosmological models 
for $k$ values; firstly, for $k \in(-\infty,-1) \cup(1 / 3, \infty)$, the deceleration parameter becomes $q<0$ and hence obeys an accelerated power law expansion; secondly, for $k \in(-1,1 / 3)$, the deceleration parameter becomes $q>0$, and therefore a decelerated expansion occurs; thirdly, for the $k=1 / 3$, the deceleration parameter becomes $q=0$, and this case corresponds to a uniformly (i.e., monotonically) expanding universe model. Also, for $k=1 / 3$, we have

$$
a(t)=\frac{I_{1}}{4 f_{0} \sqrt{\Psi_{0}}} t+a_{0} .
$$

This solution, corresponding to a universe with the pressureless Dirac field, is similar to the matter (or dust) dominant universe in the standard $2+1$ dimensional general relativity [70]. Therefore, this solution shows that the Dirac field behaves as a standard pressureless matter field in the $2+1$ dimensional teleparallel gravity.

Furthermore, we can define the equation of state parameter of the fermionic field by using the energy density (26) and pressure (23) as $\omega_{f}=p_{f} / \rho_{f}$ to search whether the fermionic field can provide alternative for dark energy or not. Given (36), (37), (45), and (48), we obtain

$$
\omega_{f}=\frac{1-3 k}{k+1}
$$

Based on the recent astrophysical observational data [71, 72], the equation of the state parameter tends to have value of -1 . Additionally, as the equation of the state parameter is less than -1 the dark energy is described by phantom, but, for $\omega \in(-1,-1 / 3)$, the quintessence dark energy is observed and the case where $\omega=-1$ corresponds to the cosmological constant. In the present work, we observed that the case where $k \in(1 / 2,1)$ corresponds to the quintessence phase, and the case where $k \in(-\infty,-1) \cup(1, \infty)$ is corresponding to the phantom phase. In both cases, the universe is both expanding and accelerating. Therefore, the results show that the fermionic field may behave like both the quintessence and the phantom dark energy field in the late-time universe.

For the case where $k=1$, the coupling and potential functions have linear forms of $\Psi$ from (36) and (37) as follows:

$$
\begin{aligned}
& F(\Psi)=f_{0} \Psi, \\
& V(\Psi)=\lambda \Psi .
\end{aligned}
$$

Therefore, without the Noether symmetry, the Friedmann equation (25) is reduced to

$$
\frac{\dot{a}}{a}-\sqrt{\frac{\lambda}{2 f_{0}}}=0,
$$

which has the following solution:

$$
a(t)=a_{0} e^{H_{0} t}, \quad \text { where } H_{0}=\sqrt{\frac{\lambda}{2 f_{0}}},
$$

where $a_{0}$ is a constant. It is clear that this solution is corresponding to the de Sitter solution that describes an inflationary epoch of the universe. Hence, it can be concluded that the Dirac field plays a role as an inflaton field in the $2+1$ dimensional teleparallel gravity. In this situation, from (26) and (23), the energy density and the pressure of the Dirac field are given by

$$
\begin{aligned}
& \rho_{f}=\frac{\lambda \Psi_{0}}{a_{0}^{2}} e^{-2 H_{0} t}, \\
& p_{f}=-\rho_{f} .
\end{aligned}
$$

Furthermore, the state parameter calculated as $\omega_{f}=-1$ corresponds to the cosmological constant.

It is interesting to note that in the case of the vanishing gauge term (i.e., $B=0$ ), the partial differential equations (32)-(33) are reduced to the equations for the usual Noether symmetry approach. In this case, in a similar form of (48), the scale factor is obtained as

$$
a(t)=\left[\widetilde{a}_{0}(k-1)\left(t-a_{1}\right)\right]^{1 /(1-k)},
$$

where $a_{1}$ is the integration constant and $\widetilde{a}_{0}=\lambda \Psi_{0}^{1-k} / 2 f_{0}$. This solution is the same as the results obtained in the context of nonminimal coupling of the fermionic field to the torsion in $3+1$ dimensional teleparallel gravity [38]. According to this solution, the deceleration parameter becomes $q=$ $-k$, and hence the universe is accelerating for $k>0$ and decelerating for $k<0$ and the case $k<0$ corresponds to uniformly expanding universe model. Also, the equation of state parameter, $\omega$, is calculated as $\omega=-k$, so that the cases where $k \in(1 / 3,1), k \in(1, \infty)$, and $k=1$ correspond to the quintessence phase, to the phantom phase, and to the cosmological constant, respectively. Hence, we can say that the fermionic field causes the same physical effect on the universe's history in both $2+1$ and $3+1$ dimensional teleparallel gravity theories under the Noether symmetry condition. On the other hand, there is a similar equivalence between the $2+1$ [35] and 3+1 [37] dimensional Einstein gravities under Noether symmetry approach in the presence of the nonminimal coupling of the fermionic field to curvature scalar.

On the other hand, the minimal coupling case is characterized by $F^{\prime}=0$, that is, by constant coupling function $F=n$. If we choose $n=1 / 2$ and ignore the gauge term (i.e., $B=0$ ), the solutions of the partial differential equations (32) are

$$
\begin{aligned}
& \alpha=\alpha_{0}, \\
& \beta_{j}=\left(\epsilon_{j} \beta_{0}-\frac{\alpha_{0}}{a}\right) \psi_{j}, \\
& \gamma_{j}=\left(-\epsilon_{j} \beta_{0}-\frac{\alpha_{0}}{a}\right) \psi_{j}^{\dagger}
\end{aligned}
$$

and from the (33) the self-interaction potential is determined as

$$
V(\Psi)=V_{0} \Psi
$$

Hence, from (22) and (25), the scale factor is calculated as

$$
a(t)=\sqrt{V_{0} \Psi_{0}} t+a_{0} .
$$


According to (58), the deceleration parameter becomes $q=0$. Hence, we can say that, in the minimal coupling case, the universe in the $2+1$ dimensional teleparallel gravity in the presence of the Dirac field is expanding uniformly, only. Furthermore, this solution describes a universe dominated by pressureless matter (or dust) [70]. Also, this solution is equivalent to the solution of the $2+1$ Einstein general relativity in the case of the minimal coupling of fermionic fields with curvature scalar [35]. On the other hand, in the nonminimal coupling case, $2+1$ dimensional teleparallel gravity has similar physical results about the history of the universe as that of $3+1$ dimensional teleparallel gravity [38], whereas it is different from the $3+1$ dimensional standard Einstein theory, because the $3+1$ dimensional standard Einstein theory explains the early-time inflation period only [37] but, as shown in this study, $2+1$ dimensional teleparallel gravity explains both the early-time and late-time inflation periods.

\section{Concluding Remarks}

The investigations to obtain suitable components that can explain the early-time inflation and late-time acceleration epoch of the universe are one of the main topics of modern cosmology. In this context, we consider the nonminimal coupling of the Dirac field to torsion in the context of teleparallel theory of gravity in $2+1$ dimensional Weitzenbock space-time to investigate whether Dirac fields may be responsible for the early-time inflation and late-time acceleration. Therefore, the identification of the nonminimal coupling and the selfinteraction functions is performed by using the Noether symmetry approach with and without a gauge term. From this approach, the obtained results can be summarized as follows:

(i) For the case where $k=1$, the scale factor obtained in (53) is $a(t)=a_{0} e^{H_{0} t}$, indicating the inflation epoch of the universe. Also this solution corresponds to the vacuum energy which is characterized by cosmological constant.

(ii) The case where $k \in(1 / 2,1)$ conforms to the quintessence dark energy component that is used to explain the late-time acceleration.

(iii) The case where $k \in(-\infty,-1) \cup(1, \infty)$ corresponds to the phantom dark energy component. This component is also used to explain the late-time acceleration.

(iv) The cosmological solution for $k=1 / 3$ corresponds to a uniformly (i.e., monotonically,) expanding universe model. Also, it obtained a solution corresponding to the matter (or dust) dominant universe with the pressure of the Dirac field $p_{f}=0$ as in the $2+1$ dimensional general relativity [70]. Hence, it shows that the Dirac field behaves as a standard pressureless matter field in the $2+1$ dimensional teleparallel gravity.

(v) Under the usual Noether symmetry condition (i.e., $B=0)$, the scale factor $a(t)$ is computed as $a(t)=$ $\left[\tilde{a}_{0}(k-1)\left(t-a_{1}\right)\right]^{1 /(1-k)}$, where $a_{1}$ is the integration constant and $\tilde{a}_{0}=\lambda \Psi_{0}^{1-k} / 2 f_{0}$. It is interesting to note that this solution is the same as the results obtained in the context of nonminimal coupling of the fermionic field to the torsion in $3+1$ dimensional teleparallel gravity [38]. Hence, we can say that, in the presence of the fermionic field, the $2+1$ dimensional teleparallel gravity has similar physical results about the evolution of the universe as that of the $3+1$ dimensional teleparallel gravity thanks to the Noether symmetry.

(vi) In the case of the minimal coupling (i.e., $F=1 / 2$ ) and under the usual Noether symmetry condition (i.e., $B=0$ ) our solutions are reduced to $2+1$ dimensional Einstein general relativity solutions, as well.

(vii) It is important to emphasize that for the nonminimal coupling and under the Noether symmetry approach with a gauge term, the $2+1$ dimensional Einstein gravity [35] has similar physical results about the history of the universe as that of both $2+1$ and $3+1$ dimensional teleparallel gravities [38] in the presence of the fermionic field.

Finally, it can be said that the Dirac field is an inflaton describing the acceleration of the universe both in the earlytime epoch and in the late-time epoch.

\section{Competing Interests}

The authors declare that there is no conflict of interests regarding the publication of this paper.

\section{Acknowledgments}

The authors thank Dr. Yusuf Kucukakca for useful discussion. This work was supported by the Scientific Research Projects Unit of Akdeniz University.

\section{References}

[1] A. H. Guth, "Inflationary universe: a possible solution to the horizon and flatness problems," Physical Review D, vol. 23, no. 2, pp. 347-356, 1981.

[2] A. D. Linde, "Chaotic inflation," Physics Letters B, vol. 129, no. 3-4, pp. 177-181, 1983.

[3] S. Perlmutter, G. Aldering, G. Goldhaber et al., "Measurements of $\Omega$ and $\Lambda$ from 42 high-redshift supernovae," The Astrophysical Journal, vol. 517, no. 2, pp. 565-586, 1999.

[4] A. G. Riess, A. V. Filippenko, P. Challis et al., "Observational evidence from supernovae for an accelerating universe and a cosmological constant," The Astronomical Journal, vol. 116, no. 3, pp. 1009-1038, 1998.

[5] E. J. Copeland, A. R. Liddle, and D. Wands, "Exponential potentials and cosmological scaling solutions," Physical Review D, vol. 57, no. 8, pp. 4686-4690, 1998.

[6] P. J. E. Peebles and B. Ratra, “The cosmological constant and dark energy," Reviews of Modern Physics, vol. 75, no. 2, pp. 559606, 2003.

[7] J. Kratochvil, A. Linde, E. V. Linder, and M. Shmakova, “Testing the cosmological constant as a candidate for dark energy," 
Journal of Cosmology and Astroparticle Physics, vol. 2004, no. 7, article no. 001, 2004.

[8] R. R. Caldwell, R. Dave, and P. J. Steinhardt, "Cosmological imprint of an energy component with general equation of state," Physical Review Letters, vol. 80, no. 8, pp. 1582-1585, 1998.

[9] V. Faraoni, "Inflation and quintessence with nonminimal coupling," Physical Review D, vol. 62, no. 2, Article ID 023504, 2000.

[10] S. Nojiri and S. D. Odintsov, "Quantum de Sitter cosmology and phantom matter," Physics Letters B, vol. 562, no. 3-4, pp. 147-152, 2003.

[11] G. W. Gibbons, "Phantom Matter and the Cosmological Constant," https://arxiv.org/abs/hep-th/0302199.

[12] A. Einstein, "Riemannian geometry with preservation of the concept of distant parallelism," Physikalisch-Mathematische Klasse, Sitzungsberichte, pp. 217-221, 1928.

[13] R. Weitzenbock, Invariance Theorie, Nordhoff, Groningen, The Netherlands, 1923.

[14] F. W. Hehl, J. D. McCrea, E. Mielke, and Y. Ne'eman, "Metricaffine gauge theory of gravity: field equations, Noether identities, world spinors, and breaking of dilation invariance," Physics Reports A, vol. 258, no. 1-2, pp. 1-171, 1995.

[15] R. Aldrovandi and J. G. Pereira, Teleparallel gravity, vol. 173 of Fundamental Theories of Physics, Springer Netherlands, 2013.

[16] K. Hayashi and T. Shirafuji, "New general relativity," Physical Review D, vol. 19, no. 12, pp. 3524-3553, 1979.

[17] J. W. Maluf and F. F. Faria, "Teleparallel gauge theory of gravity," Annalen der Physik, vol. 524, no. 6-7, pp. 366-370, 2012.

[18] S. Giddings, J. Abbott, and K. Kuchař, "Einstein's theory in a three-dimensional space-time," General Relativity and Gravitation, vol. 16, no. 8, pp. 751-775, 1984.

[19] M. Bañados, C. Teitelboim, and J. Zanelli, "Black hole in threedimensional spacetime," Physical Review Letters, vol. 69, no. 13, pp. 1849-1851, 1992.

[20] E. A. Bergshoeff, O. Hohm, and P. K. Townsend, "Massive gravity in three dimensions," Physical Review Letters, vol. 102, no. 20, Article ID 201301, 2009.

[21] G. Clément, "Warped $\mathrm{AdS}_{3}$ black holes in new massive gravity," Classical and Quantum Gravity, vol. 26, no. 10, Article ID 105015, 2009.

[22] G. Gecim and Y. Sucu, "Tunnelling of relativistic particles from new type black hole in new massive gravity," Journal of Cosmology and Astroparticle Physics, vol. 2013, no. 2, article no. 023, 2013.

[23] G. Gecim and Y. Sucu, "Dirac and scalar particles tunnelling from topological massive warped-AdS 3 black hole," Astrophysics and Space Science, vol. 357, article 105, 2015.

[24] S. Deser, R. Jackiw, and S. Templeton, "Three-dimensional massive gauge theories," Physical Review Letters, vol. 48, no. 15, pp. 975-978, 1982.

[25] A. N. Aliev and Y. Nutku, "A theorem on topologically massive gravity," Classical and Quantum Gravity, vol. 13, no. 3, pp. L29L32, 1996.

[26] K. Ait Moussa, G. Clément, and C. Leygnac, "The black holes of topologically massive gravity," Classical and Quantum Gravity, vol. 20, no. 24, pp. L277-L283, 2003.

[27] T. Kawai, "Teleparallel theory of $(2+1)$-dimensional gravity," Physical Review D, vol. 48, no. 12, pp. 5668-5675, 1993.

[28] T. Kawai, "Generators of internal Lorentz transformations and of general affine coordinate transformations in teleparallel theory of $(2+1)$-dimensional gravity," Progress of Theoretical Physics, vol. 94, no. 5, pp. 915-929, 1995.
[29] T. Kawai, "Black-hole-type solution in teleparallel theory of (2+1)-dimensional gravity", Progress of Theoretical Physics, vol. 94, no. 6, pp. 1169-1174, 1995.

[30] M. Blagojević and B. Cvetković, "Siklos waves with torsion in 3D," Journal of High Energy Physics, vol. 2014, no. 11, article no. 141, 2014.

[31] A. A. Sousa and J. W. Maluf, "Black holes in $2+1$ teleparallel theories of gravity," Progress of Theoretical Physics, vol. 108, no. 3, pp. 457-470, 2002.

[32] R. Ferraro and F. Fiorini, "Spherically symmetric static spacetimes in vacuum $\mathrm{f}(\mathrm{T})$ gravity," Physical Review $D$, vol. 84 , no. 8 , Article ID 083518, 2011.

[33] P. A. González, E. N. Saridakis, and Y. Vásquez, "Circularly symmetric solutions in threedimensional teleparallel, $\mathrm{f}(\mathrm{T})$ and Maxwell-f(T) gravity," Journal of High Energy Physics, vol. 2012, no. 7, article no. 053, 2012.

[34] P. A. González, J. Saavedra, and Y. Vásquez, "Three-dimensional hairy black holes in teleparallel gravity," Astrophysics and Space Science, vol. 357, article 143, 2015.

[35] G. Gecim, Y. Kucukakca, and Y. Sucu, "Noether gauge symmetry of dirac field in (2+1)-dimensional gravity," Advances in High Energy Physics, vol. 2015, Article ID 567395, 7 pages, 2015.

[36] B. Saha, "Spinor model of a perfect fluid and their applications in Bianchi type-I and FRW models," Astrophysics and Space Science, vol. 331, no. 1, pp. 243-255, 2011.

[37] R. C. De Souza and G. M. Kremer, "Noether symmetry for non-minimally coupled fermion fields," Classical and Quantum Gravity, vol. 25, no. 22, Article ID 225006, 2008.

[38] Y. Kucukakca, "Teleparallel dark energy model with a fermionic field via Noether symmetry," The European Physical Journal C, vol. 74, article 3086, 2014.

[39] F. W. Hehl and B. K. Datta, "Nonlinear spinor equation and asymmetric connection in general relativity," Journal of Mathematical Physics, vol. 12, no. 7, pp. 1334-1339, 1971.

[40] F. W. Hehl, "Spin and torsion in general relativity: I. Foundations," General Relativity and Gravitation, vol. 4, no. 4, pp. 333349, 1973.

[41] F. W. Hehl, "Spin and torsion in general relativity II: geometry and field equations," General Relativity and Gravitation, vol. 5, no. 5, pp. 491-516, 1974.

[42] L. H. Ryder and I. L. Shapiro, "On the interaction of massive spinor particles with external electromagnetic and torsion fields," Physics Letters A, vol. 247, no. 1-2, pp. 21-26, 1998.

[43] H. I. Arcos and J. G. Pereira, "Torsion gravity: a reappraisal," International Journal of Modern Physics D, vol. 13, no. 10, pp. 2193-2240, 2004.

[44] V. C. de Andrade, L. C. Guillen, and J. G. Pereira, "Teleparallel spin connection," Physical Review D, vol. 64, no. 2, Article ID 027502, 2001.

[45] F. W. Hehl, P. Von der Heyde, G. D. Kerlick, and J. M. Nester, "General relativity with spin and torsion: foundations and prospects," Reviews of Modern Physics, vol. 48, no. 3, pp. 393416, 1976.

[46] V. De Sabbata and M. Gasperini, Introduction to Gravitation, World Scientific, Singapore, 1985.

[47] V. C. de Andrade and J. G. Pereira, "Riemannian and teleparallel descriptions of the scalar field gravitational interaction," General Relativity and Gravitation, vol. 30, no. 2, pp. 263-273, 1998.

[48] J. W. Maluf, "Dirac spinor fields in the teleparallel gravity: comment on 'Metric-affine approach to teleparallel gravity", Physical Review D, vol. 68, no. 10, Article ID 108501, 2003. 
[49] D. Griffiths, Introduction to Elementary Particles, Wiley-VCH Pub, 2008.

[50] Y. Sucu and N. Ünal, "Exact solution of Dirac equation in $2+1$ dimensional gravity," Journal of Mathematical Physics, vol. 48, no. 5, Article ID 052503, 2007.

[51] W. G. Unruh and P. Newbury, "Solution to $2+1$ gravity in the drebein formolism," Physical Review D, vol. 48, no. 6, pp. 26862701, 1993.

[52] O. Unver and O. Gurtug, "Quantum singularities in $(2+1)$ dimensional matter coupled black hole spacetimes," Physical Review D, vol. 82, no. 8, Article ID 084016, 2010.

[53] J. L. Boldo, J. A. Helayël-Neto, and N. Panza, "Propagating torsion in 3D-gravity and dynamical mass generation," Classical and Quantum Gravity, vol. 19, no. 8, pp. 2201-2209, 2002.

[54] G. de Berredo-Peixoto, "On the static solutions in gravity with massive scalar field in three dimensions," Classical and Quantum Gravity, vol. 29, no. 19, p. 4305, 2003.

[55] J. B. Formiga, "Comment on 'Metric-affine approach to teleparallel gravity," Physical Review D, vol. 88, no. 6, Article ID 068501, 2013.

[56] E. W. Mielke, "Consistent coupling to Dirac fields in teleparallelism: comment on 'Metric-affine approach to teleparallel gravity,' Physical Review D, vol. 69, no. 12, Article ID 128501, 2004.

[57] Y. N. Obukhov and J. G. Pereira, "Lessons of spin and torsion: reply to 'Consistent coupling to Dirac fields in teleparallelism,"' Physical Review D, vol. 69, no. 12, Article ID 128502, 2004.

[58] M. Demiański, R. de Ritis, G. Marmo et al., "Scalar field, nonminimal coupling, and cosmology," Physical Review D, vol. 44, no. 10, pp. 3136-3146, 1991.

[59] S. Capozziello and R. de Ritis, "Relation between the potential and nonminimal coupling in inflationary cosmology," Physics Letters A, vol. 177, no. 1, pp. 1-7, 1993.

[60] V. I. Arnold, Mathematical Methods of Classical Mechanics, Springer, New York, NY, USA, 1989.

[61] E. Noether, "Invariante variationsprobleme," Nachrichten von der Gesellschaft der Wissenschaften zu Göttingen, MathematischPhysikalische Klasse, vol. 1918, pp. 235-257, 1918.

[62] H. Wei, X.-J. Guo, and L.-F. Wang, "Noether symmetry in $\mathrm{f}(\mathrm{T})$ theory," Physics Letters B, vol. 707, no. 2, pp. 298-304, 2012.

[63] T. Feroze and I. Hussain, "Noether symmetries and conserved quantities for spaces with a section of zero curvature," Journal of Geometry and Physics, vol. 61, no. 3, pp. 658-662, 2011.

[64] M. Tsamparlis and A. Paliathanasis, "Three-fluid cosmological model using Lie and Noether symmetries," Classical and Quantum Gravity, vol. 29, no. 1, Article ID 015006, 2012.

[65] S. Basilakos, S. Capozziello, M. De Laurentis, A. Paliathanasis, and M. Tsamparlis, "Noether symmetries and analytical solutions in $\mathrm{f}(\mathrm{T})$ cosmology: a complete study," Physical Review D, vol. 88, no. 10, Article ID 103526, 2013.

[66] A. Paliathanasis, S. Basilakos, E. N. Saridakis et al., "New Schwarzschild-like solutions in $\mathrm{f}(\mathrm{T})$ gravity through Noether symmetries," Physical Review D, vol. 89, no. 10, Article ID 104042, 2014.

[67] G. W. Bluman and S. Kumei, Symmetries and Differential Equations, Springer, New York, NY, USA, 1989.

[68] H. Stephani, Differential Equations; Their Solutions Using Symmetries, Cambridge University Press, New York, NY, USA, 1989.

[69] A. Paliathanasis and M. Tsamparlis, "Two scalar field cosmology: conservation laws and exact solutions," Physical Review D, vol. 90, no. 4, Article ID 043529, 2014.
[70] J. D. Barrow, D. J. Shaw, and C. G. Tsagas, "Cosmology in three dimensions: steps towards the general solution," Classical and Quantum Gravity, vol. 23, no. 17, pp. 5291-5321, 2006.

[71] P. A. R. Ade, N. Aghanim, C. Armitage-Caplan et al., "Planck 2013 results. XVI. Cosmological parameters," Astronomy and Astrophysics, vol. 571, article A16, 2014.

[72] S. Kumar and L. Xu, "Observational constraints on variable equation of state parameters of dark matter and dark energy after Planck," Physics Letters B, vol. 737, pp. 244-247, 2014. 

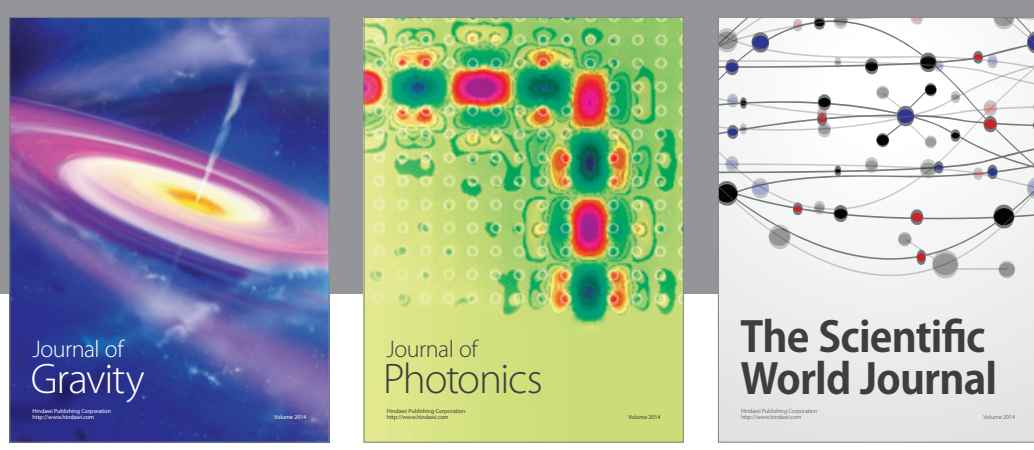

The Scientific World Journal
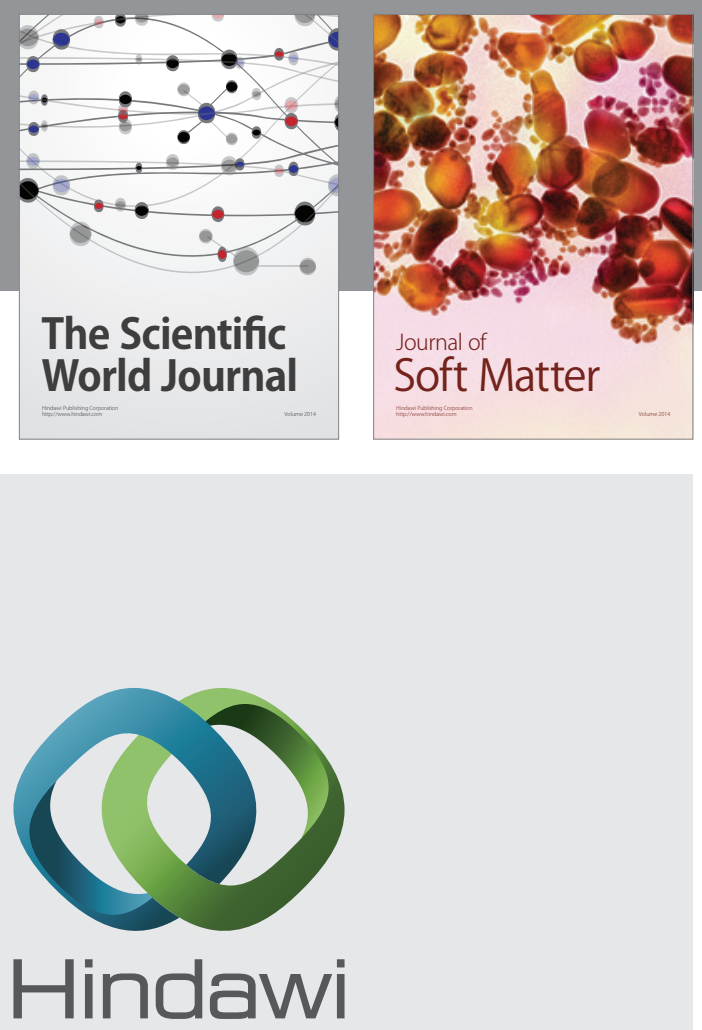

Submit your manuscripts at

https://www.hindawi.com
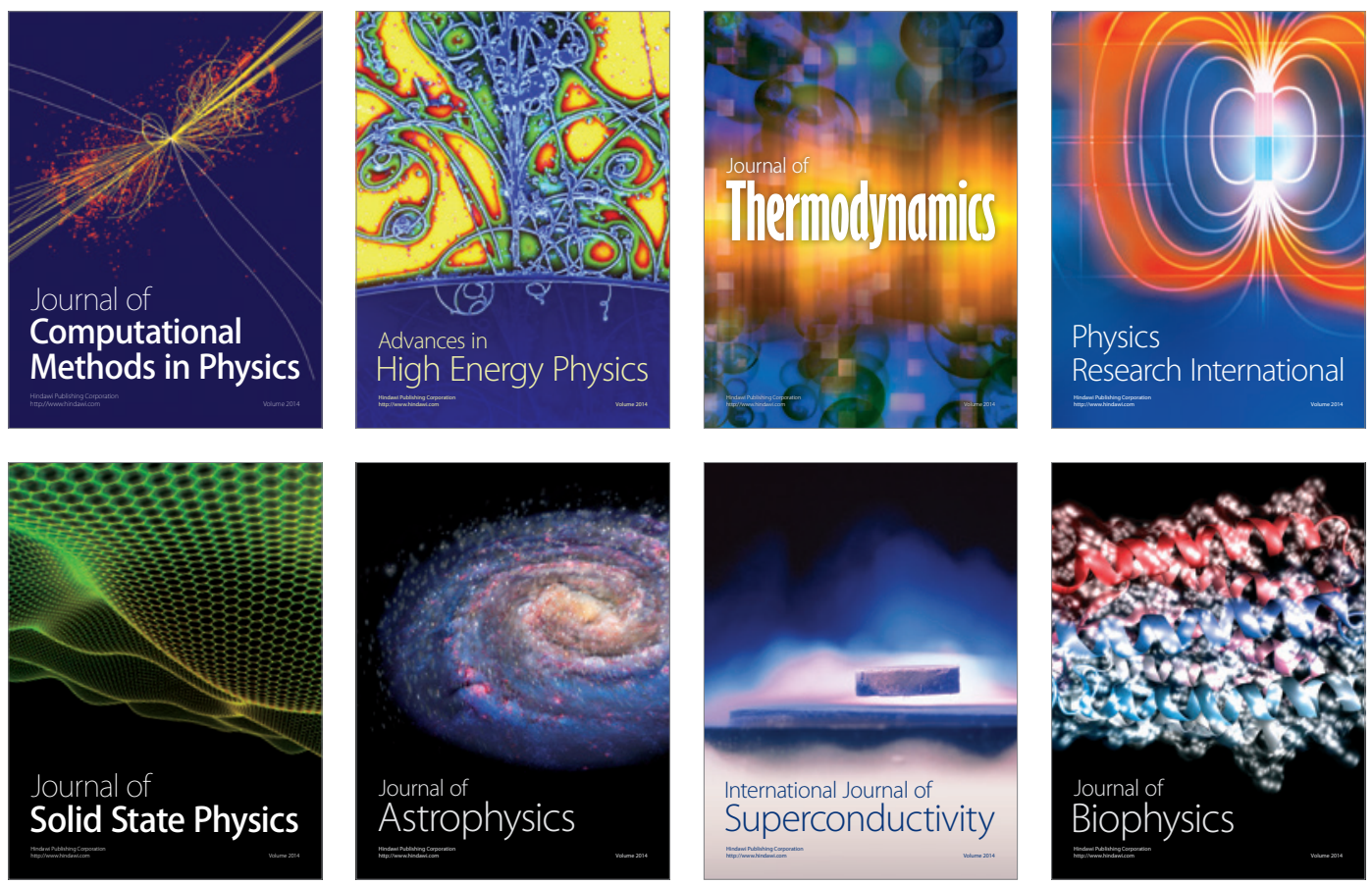
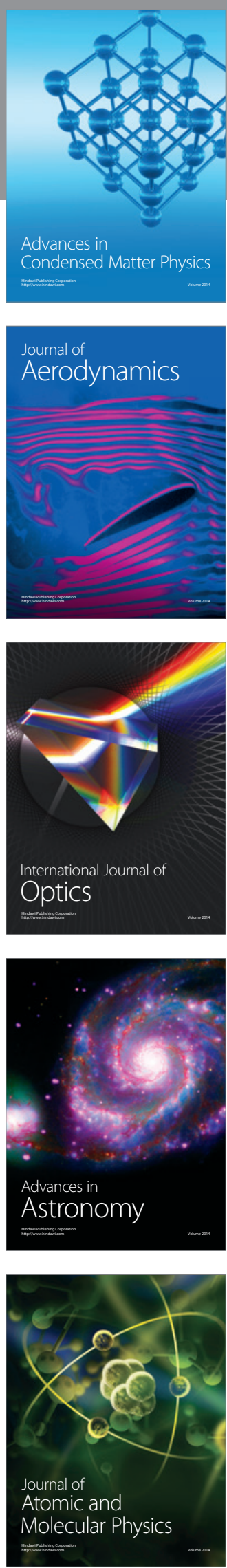